\title{
CORPORATE DOWNSIZING TO REBUILD TEAM SPIRIT: HOW COSTLY VOTING CAN FOSTER COOPERATION
}

\author{
Antonio Cabrales \\ Universidad Carlos III de Madrid and \\ CEPR
}

\author{
Antoni Calvó-Armengol \\ ICREA, Universitat Autònoma de \\ Barcelona and CEPR
}

\begin{abstract}
We propose a new mechanism to achieve coordination through voting, for which we discuss a number of real-life applications. Among them, the mechanism provides for a new theory for downsizing in organizations. A crisis may lead to a decrease in the willingness to cooperate in an organization, and therefore to a bad equilibrium. A consensual downsizing episode may signal credibly that survivors are willing to cooperate, and thus, it may be optimal and efficiency-enhancing (for the individuals remaining in the organization), as the empirical evidence suggests. A variation of the same mechanism leads to "efficient" upsizing. (JEL: C70, D70, M50)
\end{abstract}

\section{Introduction}

It is often the case that a group can improve upon a dysfunctional status quo if its members undertake activities with positive feedback loops. That is, they must engage in activities such that the profitability of an action increases when others are also taking it (also known as actions with strategic complementarities). ${ }^{1}$ This kind of situation normally leads to multiple equilibria. Therefore, improving upon the status quo requires that agents align expectations about others' actions in the right way, so that the improving equilibrium is indeed implemented.

The aim of this paper is to describe a mechanism that guarantees that expectations are correctly aligned, and that we argue is pervasive in the real world. The mechanism requires that agents facing a bad status quo collectively approve an action which is costly for the whole group. This action only yields benefits in the

Acknowledgments: We gratefully acknowledge the financial help from Fundacion BBVA, from the Spanish Ministry of Science and Technology and FEDER under grants CONSOLIDFR-INGENIO 2010(CSD2006-00-16), SEJ200508783-C04-01, SEC2003-03474, and SEJ2005-01481ECON, and from the Barcelona Economics Program of XREA. We thank Gary Charness, Matt Jackson, Joel Shapiro, Joel Sobel, Fernando Vega-Redondo, and various seminar audiences for their helpful comments. The usual disclaimer applies.

E-mail addresses: Cabrales: antonio.cabrales@uc3m.es; Calvó-Armengol: antoni.calvo@uab.es

1. See Vives (2005) for a recent exhaustive account of new developments on games with complementarities. 
game with strategic complementarities if the right equilibrium is implemented. Thus, only agents who intend to implement the improving equilibrium will support such action. It is the act of voting on a costly action that operates as an equilibrium selection device by providing a credible behavioral signal of the intention to cooperate.

There are a number of real-life phenomena that can be accounted for by this costly voting mechanism combined with an ensuing coordination game. These range from firms' downsizing practices to scapegoating, among others. Although we focus on corporate downsizing in our analysis, we first briefly describe some alternative applications of the mechanism.

An episode in the conquest of Mexico in the early sixteenth century offers a first example. In 1519, Hernán Cortés and 600 Spaniards landed on the coast of Mexico. The ships that brought them from Cuba were then purposefully destroyed. According to Bernal Díaz del Castillo (1984, ch. 58), who accompanied Cortés in his trip, this was done as a means to avoid "occasions for internal dissension" during the battles to follow against the Aztec empire. ${ }^{2}$ Díaz del Castillo, in fact, says that although Cortés had already thought about this, he wanted the initiative on this measure to come from his men. ${ }^{3}$ Destroying the ships is, clearly, a costly action, and the ensuing victorious military campaign can be read as a sign of good internal harmony and coordination among the Spanish troops. ${ }^{4}$ Going from the particular to the general, most constitutions give the power to declare war to the legislative, rather than the executive, branch of government. For example, Article 1, section 8, of the American Constitution states, "The Congress shall have Power to declare war," even though Article 2, section 2, says, "the President shall be Commander in Chief of the Army and Navy of the United States." 5

The critical account of scapegoat episodes throughout both history and literature by Girard (1982) offers another example of this mechanism at play. ${ }^{6}$ Girard starts by noticing that prior to the scapegoat episode there is often a crisis and a breakdown of cooperation in the societies where they occur. The scapegoat

2. "Y no quedasen ocasiones, porque entre tanto que estábamos la tierra adentro no se alzasen otras personas como los pasados." (ch. 58, p. 215, emphasis in the original).

3. "El mismo Cortés lo tenía ya concertado, sino que quiso que saliese de nosotros." The extent to which the decision to destroy the ships was indeed collectively settled is debated (Reynolds 1959). For our purposes, it suffices that enough people have thought that both the action and consultation with the men were a good idea.

4. This historical episode has been interpreted as an example of the value of commitment (Schelling 1960). In our view, without consultation there is still a bad equilibrium where the ship is not destroyed, as coordination is not expected after the destruction. So we argue that it is the combination of a bad outcome and consultation that turns weakness to strength.

5. Just to provide two more examples, the present Spanish Constitution (Article 63.3), as well as the French one (Article 35), also require direct parlimentary approval for the declaration of war.

6. As a matter of fact, a good deal of inspiration for our paper (and the starting point for this research) was obtained by reading Girard (1982). 
episode, he argues, serves then as a way for society to regain cooperation. The collective dimension of scapegoating episodes together with the arbitrary nature of this act of violence corresponds to the costly voting stage of our model that helps to implement the desirable cooperative outcome. Girard also notices that scapegoats are often characterized by a mark, which distinguishes them for the rest of society. This differentiating mark plays the role of a focal point that serves to coordinate on them as the victims, something missing from our model (because it is unnecessary).

The application of this mechanism goes beyond these, admittedly important, sociopolitical examples. We now turn our attention to a clearly economic phenomenon, which will be the focus for the remainder of this paper. This phenomenon is the reduction in the number of members of the organization, also called downsizing.

In this paper we propose a new theory to explain downsizing, based on the mechanism previously outlined, whereby organization members vote and then undertake a costly action, followed by a coordination game.

Many activities within firms involve strategic complementarities. As Milgrom and Roberts (1995) argue, these complementarities are one of the main reasons for the existence of firms. ${ }^{7}$ Suppose that, for some reason, members of an organization start to entertain doubts about the ability to coordinate of some of its fellow members. Then, it is rational for everybody in the organization to undertake any action whose payoff is less sensitive to coordinating with others. As a result, productivity decreases and there is a common loss. An obvious solution to this corporate problem is to expel the initiators of this negative feedback loop. This by itself would be a reason for downsizing. But notice that in the critical stage no one in the organization is cooperating, so the parties initiating the loss of coordination may not be easy to locate. They are simply doing what everybody else does. And even if the initiators of the loss of cooperation were easy to locate, there is no guarantee that, after reorganization, the remaining members would in fact cooperate again, as expectations need not be aligned any more.

We call strategic downsizing a process by which the organization collectively decides whether to implement a restructuring plan. We show that strategic downsizing can restore cooperation in the streamlined organization. This might occur even if the individuals who are laid-off are not the initiators of the breakdown in coordination. Our result relies on two crucial assumptions: reorganization can be costly, but it can be profitable as well. ${ }^{8}$ We discuss costs and benefits separately.

7. "[S]trong complementarities make it more likely that $[\ldots]$ central strategic direction will be valuable" (Milgrom and Roberts 1995, p. 190). Kreps (1990) has also argued that the alignment of expectations is crucial in organizations, and may be the rationale for a "corporate culture." See also Crémer (1993) and Lazear (1995) on other perspectives of corporate culture.

8. In the remainder of this paper, when we talk about the benefits of corporate downsizing, we refer to the benefits accruing to those individuals who remain a part of the organization after downsizing. 
Let us first focus on the possibility for gains, and why they cannot be realized without downsizing. In our game a cooperative action can be individually profitable, but this is true only if most people also take that action. Suppose now that a shock to the economy, sector, or firm opens new possibilities outside of the organization for some of its members. ${ }^{9}$ These people are unwilling to coordinate with the rest of the group, so cooperation is doomed as the size of the organization becomes large. Indeed, the probability that not enough members take the cooperative action becomes very large. However, in a smaller organization, the probability of not encountering enough cooperators can be sufficiently reduced so that cooperation is viable. Downsizing is, thus, necessary for cooperation.

Note, however, that a firm may do the reorganization and find itself in a bad equilibrium anyway. Here is where the potential cost for downsizing becomes important. Suppose that, besides the potential benefit previously described from potential cooperation, downsizing has a certain cost. For instance, there may be increasing returns to scale in organization size. Assume that the members of the organization are consulted on whether the reorganization should take place. Because downsizing without cooperation is assumed to be costly, supporting the reorganization is only reasonable for a member who plans to cooperate after reorganization. Thus, the approval of downsizing is a signal that its supporters are ready to cooperate in the streamlined organization. This is the mechanism that coordinates expectation, and thus ensures coordination. In a sense, the public approval and the potential cost of downsizing are a sufficient condition for cooperation.

We also show that, besides its ability to achieve coordination through downsizing, a similar mechanism of collective approval can lead to "efficient" upsizing.

At this point, it is worth reviewing some stylized facts on downsizing. Oulton (2000) shows that the productivity is negatively correlated with size changes in a study of British manufacturing firms. Foster, Haltinwanger, and Krizan (2001) has the same type of finding for American retail-trade firms. A natural explanation for this would be technological. If technology has decreasing returns to scale, downsizing would imply a gain in productivity. For this reason, it is interesting to turn to a study by Barnes and Haskel (2001, Table 4) for a sample of British manufacturing firms since they decompose the effect of productivity between upsizing and downsizing firms. They show that, for firms that downsize, the proportion whose productivity increases can be between 2 and 5 times higher than those whose productivity decreases depending on the period. Interestingly for us, the same qualitative finding is true for firms that upsize. ${ }^{10}$ It is important also

9. For instance, they may start an active on-the-job search.

10. Baily, Bartlesman, and Haltiwanger (1996) analyze plant level data in the U.S. manufacturing sector and conclude that "increased employment as well as productivity contribute almost as much to overall productivity growth in the 1980 s as the plants that increased productivity at the expense of employment" (p. 1). 
to note that not all downsizing is a response to decreases in demand (Chadwick, Hunter, and Walston 2004). ${ }^{11}$ This body of evidence is consistent with a picture of firms mostly in the increasing returns part of the cost curve that downsize to recover coordination, as we posit in our model.

A large amount of literature in management deals with the costs and benefits of downsizing. There is no widespread consensus, neither from the theoretical nor from the empirical perspective, on whether the actual effects of downsizing on productivity are positive or negative. For example, on the positive side Theorell et al. (2003) show that the rate of long-term sick leave, a key productivity indicator, decreased after downsizing in Swedish hospitals. Amabile and Conti (1999), on the other hand, show that downsizing is often detrimental for creativity, although this depends on the circumstances and the negative effects typically decrease over time.

The efficiency-inducing properties of voting in our mechanism are also supported in the available data. Bassi and Van Buren (1997) show evidence that "involving employees in a downsizing process tend to produce positive outcomes" (p. 39). Also, management advisors typically recommend to effectuate a restructuring in a transparent and consensual manner (see, e.g., Borgen 2000). In most European countries it is mandatory to consult with workers when developing downsizing plans. ${ }^{12}$

Our mechanism for equilibrium switching requires that agents avoid dominated strategies, and that they realize that others will do so as well. It is legitimate to question whether this selection process is empirically relevant. The answer is likely to be positive. On the one hand, only two rounds of deletion of dominated strategies are required. This does not seem to pose such stringent demands on the common knowledge of rationality. Furthermore, and very importantly, the available experimental evidence is mostly positive for games that share similar features with ours. The equilibrium selection problem can be divided, in a broad sense, between games where there is no distributional conflict (the equilibria are Pareto-ranked) and those games where different agents prefer different equilibria. In our case, the agents agree on which is the desirable equilibrium outcome. It turns out that the available experimental evidence in games that share this feature supports the behavioral relevance of forward induction (see, e.g., Van Huyck, Battalio, and Beil 1993; Broseta, Fatás and Neugebauer 2003; and the discussion in Section 3 of Brandts, Cabrales, and Charness 2006).

11. "Firms, however, may also reduce jobs even in environments in which demand is robust, seeking increased operating efficiencies" (Chadwick, Hunter, and Walston 2004, p. 405).

12. For example, in the U.K., "[I]n non-unionised sectors, those with more than 20 employees must embark on a 30-day consultation with a body of elected workers' representatives. Companies with more than 100 employees require a 90-day consultation period." Financial Times (London), October 28, 2002, p. 20. 
The mechanism that induces cooperation in our paper is connected to forward induction, as in the papers of Van Damme (1989) and Ben-Porath and Dekel (1992). In Ben-Porath and Dekel, the potential for self-sacrifice is sufficient to obtain the desired outcome, whereas in our game the sacrifice has to be effective. To understand this difference, let us sketch the argument in Ben-Porath and Dekel. They consider a coordination game where, in addition, one player (and only one) is given the possibility to undertake a costly action (call it "burning money"). For this player, burning money and then playing the action leading to his worst equilibrium outcome is weakly dominated. Thus, burning money signals the intention to obtain his favorite equilibrium. But then, even if he does not burn money, he will obtain his favorite equilibrium payoff (as otherwise he would burn money). In our model, in the original organization, and given the players' beliefs, only one equilibrium outcome can be sustained. Our costly action (downsizing) does not guarantee per se a better equilibrium (unlike in Ben-Porath and Dekel). However, if the organization is downsized, for the same beliefs, it now faces an equilibrium selection problem. The (costless) collective choice procedure then solves the equilibrium selection problem with forward-induction type arguments (voting yes and then not cooperating is weakly dominated). So, in our model, the effective sacrifice creates an equilibrium selection problem, which is solved by bringing the sacrifice to collective approval. In Ben-Porath and Dekel, on the other hand, the equilibrium selection problem is a given, which the potential for sacrifice then solves.

Section 2 describes the model, and the results are presented in Sections 3 to 8 . Section 9 discusses the related literature and concludes.

\section{The Model}

\subsection{Productive Technology}

An organization produces output in a way that depends both on the size of the organization and its members' productive actions. We denote by $u_{i}\left(a_{1}, \ldots, a_{n} ; n\right)$ the individual payoff of a current member $i$ of an organization composed of $n$ members where member $k$ takes action $a_{k}$. This payoff equals her marginal productivity (net of effort costs). We suppose that $u_{i}$ is multiplicatively separable in the vector $\left(a_{1}, \ldots, a_{n}\right)$ of productive actions and in the size $n$ of the organization; that is,

$$
u_{i}\left(a_{1}, \ldots, a_{n} ; n\right)=h_{i}^{n}\left(a_{1}, \ldots, a_{n}\right) F(n) .
$$

The size effect function, $F(n)$, common to all organization members, is assumed to be increasing for some range $[0, \bar{n}]$. This reflects increasing returns to scale (thus increasing marginal productivity) of production output to organizational size, at least below a critical organization size. The function $h_{i}^{n}\left(a_{1}, \ldots, a_{n}\right)$ reflects 
complementarities of efforts between members of the organizations; it depends on the number of team members and on their actions profile. This is the kind of complementarity to which Kreps (1990) and Milgrom and Roberts (1995) refer.

For simplicity, there are only two productive actions for each member. The productive action space for member $i$ is $A^{i}=\{0,1\}$, where $a_{i}=1$ represents the option to contribute to an activity which enhances productivity (cooperative action), and $a_{i}=0$ represents the option not to contribute (noncooperative action). For any productive action profile $\left(a_{1}, \ldots, a_{n}\right)$, let $a=\sum_{i=1}^{n} a_{i}$ be the total number of contributors. The productive action effect varies across members in the following way:

$$
h_{i}^{n}\left(a_{i}, a_{-i}\right)= \begin{cases}\tau^{n}(a) & \text { if } a_{i}=1, \\ 1 & \text { if } a_{i}=0 .\end{cases}
$$

The individual return to choosing the noncooperative action $a_{i}=0$ is constant (and equal to one) irrespectively of the choice made by other members. ${ }^{13}$ The return to the cooperative action $a_{i}=1$ depends on the productive action profile as it varies with the total number of contributors.

The return to the cooperative action is represented by a nondecreasing function $\tau^{n}:\{1, \ldots, n\} \rightarrow \mathbb{R}$. In words, the returns to cooperation increase (weakly) with the number of cooperators. Consider now two teams of different sizes $m>n$. We further assume that $\tau^{m}(m-k) \geq \tau^{n}(n-k)$, for all $k \leq \min \{n, m\}$. In other words, given a fixed number of noncooperators $k$, the returns to cooperation are non-decreasing in team size.

\subsection{The Dilution of Team Spirit}

The team spirit of an organization is reflected in the profile $a$ of productive actions taken by its members. Organizations with a sufficiently high proportion of members choosing $a_{i}=1$ display a high team spirit, which translates into higher per capita payoffs for members building actively such team spirit.

Suppose now that, at some point in time, all members in the organization acquire the belief that, with some probability $\mu$, each member $i$ sticks to action $a_{i}=0$, irrespectively of any other factor. This may be due to a shock to the economy, sector, or firm, which changes the payoff structure outside of the organization for some of its members. Think of the cooperative action as a firm-specific task providing no benefits outside the current organization. For some members, the

13. The results would be essentially unchanged if noncooperators were able to "enjoy" part of the efforts of cooperators. That is, if we had $h_{i}^{n}\left(0, a_{-i}\right)=\underline{\tau}^{n}(a)$, with $\tau^{n}(a)$ increasing more rapidly with $a$ than $\tau^{n}(a)$, and also with $\tau^{n}(0)>\tau^{n}(0)$. A rationalization for the lower rate of increase of $\underline{\tau}^{n}(a)$ could be a probability of detection/punishment changing with $a$. 
shock could generate profitable outside opportunities. Then, for these players, the time spent undertaking the cooperative action can be more profitably used by exploring actively these outside opportunities. Formally, for all these people, the productive action effect becomes $\tilde{h}_{i}^{n}$, where $\tilde{h}_{i}^{n}\left(0, a_{-i}\right)>\tilde{h}_{i}^{n}\left(1, a_{-i}\right)$ for all $a_{-i}$. For these players, it is a dominant strategy to play $a_{i}=0$, and we set $\tilde{h}_{i}^{n}\left(0, a_{-i}\right)=h_{i}^{n}\left(0, a_{-i}\right)=1$. The probability $\mu$ is, then, the ex ante probability assigned by all individuals in the organization to this event.

We refer to the members with this kind of behavior as pathological individualists and denote the set of members with this behavior by PI. Members in PI do not take part in team building activities under any circumstances. Prior to the revelation of their private type, the expected output for a member $i$ that plans to choose action $a_{i}=1^{14}$ when every other player plans to choose $a_{i}=1$ (if in the interim they are not revealed to be a $P I$-type), is $E u_{i}=(1-\mu) H(n)+\mu F(n)$, where

$$
H(n)=F(n) \sum_{j=0}^{n-1}\left(\begin{array}{c}
n-1 \\
j
\end{array}\right) \mu^{j}(1-\mu)^{n-1-j} \tau^{n}(n-j) .
$$

\subsection{The Corporate Downsizing Game}

Initially, an organization is composed of a set $N$ of members.

The game consists of two stages. In the first stage, all organization members participate in the corporate downsizing procedure. This is a collective choice process that we model through (several variations of) a voting game. Then, at an interim stage, a nature move determines the players' types. Each organization member has a probability $\mu$ of belonging to $P I$; this is independent and identical across members. In the second stage, the remaining members in the organization choose their productive actions according to their types.

We have assumed at this point, for expositional simplicity, a common prior and revelation of information after downsizing is decided upon. This allows us to exclude the complexity of signaling-type phenomena at the downsizing stage. We later show that the basic thrust of the results still holds under a more natural informational assumption.

We now analyze the game for different scenarios about the first stage procedure. In each scenario we characterize the strategy profiles that survive two rounds of deletion of weakly dominated strategies (and thus are subgame-perfect equilibria) of the downsizing game. Let us be a bit more precise.

14. Note that, if player $i$ ends up being a pathological individualist once private types are revealed to players, she will not follow this intended plan of action and rather, given her type, select and stick to the non-cooperative alternative $a_{i}=0$. 
REMARK 1. In the remainder of the paper, statements about rounds of deletion of weakly dominated strategies mean that in every round all players simultaneously eliminate all their dominated strategies.

\section{Approving or Rejecting an Elimination}

One common procedure for corporate downsizing requires the membership to approve or reject the elimination of a certain subgroup. Here, we look at the case where the candidate subgroup is exogenously given: Section 5 considers the case where the subgroup is endogenous.

There is a candidate subgroup $C$ for elimination. Each individual $i \in N$ casts a positive or negative vote on the elimination of $C$. The choice of $C$ is exogenous to the approval procedure. Either the CEO or perhaps an outside consultant makes the proposal. The final decision is taken by $k$-majority voting, that is, for the set $C$ to be eliminated, at least $k$ players have to vote for the elimination. ${ }^{15}$

Let $c$ be the cardinality of $C$. Define also

$$
H^{*}=\min \{H(p) \mid k \leq p \leq n-c\} .
$$

Notice that this minimum value depends on the size of majority approval size $k$ and the beliefs $\mu$.

Proposition 1. Suppose that

$$
(1-\mu) H^{*}+\mu F(n-c)>F(n)>H(n) .
$$

In all strategy profiles that survive two rounds of deletion of weakly dominated strategies, all players $i \in N \backslash\{C \cup P I\}$ vote for elimination of $C$ and then choose $a_{i}=1$ after the elimination.

Proof. First, observe that for all $i \notin C$ approving the elimination and then choosing $a_{i}=0$ is weakly dominated by not approving the elimination and then choosing $a_{i}=0$. Indeed, the expected payoff for player $i \notin C$ when nobody is eliminated and she chooses $a_{i}=0$ is $F(n)$. The payoff for player $i \notin C$ when $C$ is eliminated and she chooses $a_{i}=0$ is $F(n-c)$, and $F(n-c)<F(n) .{ }^{16}$ Because casting a vote for the elimination of $C$ by any player $i \notin C$ may be pivotal for this elimination, given the requirement of $k$-majority approval, the domination follows. Thus, any player who votes for the elimination of $C$ will play $a_{i}=1$ if

15. The "yes/no" voting procedure over two alternatives we adopt here rules out issues of strategic voting or cycling patterns.

16. Note that, if in the interim stage $i \in P I$, then $a_{i}=0$ by our assumption on the behavior of pathological individualists. 
in the interim stage $i \notin P I$. Therefore, a lower bound for the expected payoff in case of elimination is given by $(1-\mu) H^{*}+\mu F(n-c)$.

Second, because $F(n)>H(n)$ is equivalent to $F(n)>(1-\mu) H(n)+$ $\mu F(n)$, it is dominant to choose $a_{i}=0$ when nobody is eliminated. The condition $(1-\mu) H^{*}+\mu F(n-c)>F(n)$ guarantees that the player $i \notin C$ prefers (ex ante) the situation where $C$ is eliminated. Because casting a vote for the elimination of $C$ by any player $i$ may be pivotal for this elimination, it is dominant to vote for this elimination (and then choose $a_{i}=1$ ).

Therefore, with exogenous candidate subgroups, downsizing is approved and members remaining in the organization (excluding pathological individualists) choose the cooperative action. Note, incidentally, that expelled members need not necessarily be pathological individualists, as this trait is not known at the time of deciding whom to exclude (or private information, in the more general setting we explore later). The collective decision to downsizing thus unfairly harms some organization members, just as scapegoats are unfairly blamed for problems of any kind. Here, as in Girard's theory, arbitrary scapegoating reestablishes cooperation.

The mechanism through which downsizing helps to enhance efficiency has two essential parts.

First, downsizing reduces the probability that an organization contains a large enough group of $P I$-types that would make cooperation impossible, even for welldisposed non- $P I$ individuals. To make this point clearer, consider the extreme case where all individuals have to cooperate in order for the "good" cooperative outcome to be effective, and that this yields very high returns $\tau^{n}(n) \gg 1$. Without downsizing, the probability of having at least one $P I$ in the organization (and thus foregoing the high payoff $\left.\tau^{n}(n)\right)$ is $1-(1-\mu)^{n}$. Suppose instead that downsizing takes place and that $c$ individuals are expelled. This probability then decreases to $1-(1-\mu)^{n-c}$, which translates into higher expected gross benefits. Of course, downsizing comes at a cost $F(n-c)-F(n)<0$. However, downsizing seems a good way to achieve efficiency (for individuals not in the expelled group) as long as this cost is small enough compared to the benefit, that is,

$$
\tau^{n}(n)\left[(1-\mu)^{n-c}-(1-\mu)^{n}\right]>F(n)-F(n-c) .
$$

Very importantly, this argument carries over to more general environments, as we show below with two examples. In particular, we do not have to assume that cooperation is rewarding only if everyone cooperates.

Second, notice that the previous argument does not guarantee that downsizing would lead to a more efficient situation. Indeed, the coordination game of the reduced organization still has multiple equilibria, and the surviving members of the downsized organization could rationally refuse to cooperate. This is where the second part of the mechanism helps. Given our assumption of increasing returns in organization size, downsizing entails a cost $F(n-c)-F(n)$ borne 
by all members of the downsized organization. Because of this cost, no rational agent would approve the downsizing if she were intending not to cooperate. So a favorable vote on downsizing acts as a credible behavioral signal that the voter intends to cooperate after reorganization takes place. Because reorganization can be beneficial if enough people cooperate, and because a positive vote signals a willingness to cooperate, everybody will approve the downsizing and non-PI members will cooperate after reorganization.

This discussion allows us to interpret the sufficient conditions for downsizing stated in Proposition 1, namely, the two inequalities in equation (2). First, at least $k$ and at most $n-c$ members have approved reorganization (or else it would not have occurred) and therefore intend to cooperate if they turn out not to be $P I$, yielding a lower bound on expected payoffs equal to $H^{*}=\min \{H(p): k \leq p \leq n-c\}$. Note that the event in which they are not $P I$ is random and this explains why the binomial terms in the expression for $H(p)$ in (1) are needed. Second, given that the individual may still turn out to be a $P I$, the lower bound in expected payoffs is a weighted sum of $H^{*}$, which bounds what she gets when not $P I$, and $F(n-c)$, which she gets when $P I$. When $(1-\mu) H^{*}+\mu F(n-c)>F(n)$, it is better to be in a downsized organization than not cooperating in the original one. Finally, the condition $F(n)>H(n)$ guarantees that members do not cooperate in the original organization.

\section{Two Classes of Coordination Payoff Functions}

We now show that the two inequalities in equation (2) can hold simultaneously for two classes of functional forms relating to coordination payoffs. We also determine the optimal size of the expelled group and work out in the examples its main comparative statics.

In what follows, we set the size effect function to $F(n)=n$.

\subsection{Threshold Games}

Suppose that the returns to coordination are represented by a nondecreasing step function such that $\tau^{n}(a)=0$ for all $a<n-\kappa$ and $\tau^{n}(n)>1$. In words, the cooperative action yields nonzero returns whenever at most $\kappa$ members in the organization choose not to contribute. The function $\tau^{n}(a)$ is nonzero for $a \in\{n-\kappa, \ldots, n\}$, a support of size $\kappa \cdot{ }^{17}$ This kind of payoff function is a generalization of the O-ring production function, originally proposed by Kremer

17. More generally, the maximum numbers of noncooperators $\kappa$ compatible with positive returns to cooperation could vary with the size $n$ of the group. All of our qualitative conclusions would still hold. 
(1993), and which has been used in a large number of empirical and theoretical works. $^{18}$

According to (1), the expected individual payoff when everybody plans to cooperate (type-permitting) are $H(n)=F(n) h(n)$, where:

$$
h(n)=\sum_{j=0}^{n-1}\left(\begin{array}{c}
n-1 \\
j
\end{array}\right) \mu^{j}(1-\mu)^{n-1-j} \tau^{n}(n-j) .
$$

We have argued above that there must be, at least, the possibility of enhanced profits for cooperation in a downsized organization. In other words, $H(n)$ must be decreasing in $n$, at least over some range. Because $F(n)$ is increasing, a necessary condition for this is that the remaining term $h(n)$ is decreasing in $n$ over some range. As established below, it turns out that a sufficient condition for this monotonicity to arise with a step function $\tau^{n}$ is $\mu n \geq \kappa$. In other words, if the expected number of $P I$-types $(\mu n)$ is larger than the maximal number $(\kappa)$ that can be present in the organization without spoiling cooperation, then a reduction in the organizational size can be beneficial.

Lemma 1. Suppose that $\mu \mathrm{n} \geq \kappa$. Then, $\mathrm{h}(\mathrm{n})$ is decreasing in $\mathrm{n}$.

Proof. Recall that, by assumption, $\tau^{n}(a)=0$ for all $a<n-\kappa$, and $\tau^{n+1}(a)=0$ for all $a<n+1-\kappa$. Then we have

$$
\begin{aligned}
h(n+1)-h(n)= & \sum_{k=0}^{\kappa}\left(\begin{array}{l}
n \\
k
\end{array}\right) \mu^{k}(1-\mu)^{n-k} \tau^{n+1}(n+1-k) \\
& \times\left[1-\frac{\left(\begin{array}{c}
n-1 \\
k
\end{array}\right)}{\left(\begin{array}{c}
n \\
k
\end{array}\right)} \frac{1}{1-\mu} \frac{\tau^{n}(n-k)}{\tau^{n+1}(n+1-k)}\right] .
\end{aligned}
$$

If the term in brackets is nonpositive for all $0 \leq k \leq \kappa$ and negative for some $0 \leq k \leq \kappa$, then $h(n)>h(n+1)$. Because

$$
\left(\begin{array}{c}
n-1 \\
k
\end{array}\right) /\left(\begin{array}{l}
n \\
k
\end{array}\right)=\frac{n-k}{n},
$$

this condition is equivalent to

$$
\mu \geq 1-\frac{n-k}{n} \frac{\tau^{n}(n-k)}{\tau^{n}(n+1-k)} \quad \text { for all } 0 \leq k \leq \kappa,
$$

18. Over 330 citations in Google Scholar on August 2006. See Heywood and Jirjahn (2004) and Mirza and Nicoletti (2004) as examples of two recent papers in very different fields who take the O-ring production function as the basis of their empirical work. 
TABLE 1. Optimal scapegoat size as a function of $n, \kappa$, and $\mu$.

\begin{tabular}{lcccccc}
\hline$(n ; \kappa)$ & $\mu=0.10$ & $\mu=0.15$ & $\mu=0.20$ & $\mu=0.25$ & $\mu=0.30$ & $\mu=0.35$ \\
\hline$(50 ; 5)$ & 7 & 22 & 24 & 24 & 24 & 24 \\
$(50 ; 10)$ & 0 & 0 & 10 & 18 & 23 & 24 \\
$(100 ; 10)$ & 20 & 47 & 49 & 49 & 49 & 49 \\
$(100 ; 15)$ & 0 & 20 & 40 & 49 & 49 & 49 \\
$(100 ; 20)$ & 0 & 0 & 20 & 36 & 46 & 49 \\
\hline
\end{tabular}

with a strict inequality for some $0 \leq k \leq \kappa$. Given that $\tau^{n}(\cdot)$ is nondecreasing in $n$, a sufficient condition for this last inequality to hold is that $\mu \geq \kappa / n$.

We now turn to the issue of what is the optimal size of the expelled group for the class of step functions. More precisely, suppose that the decision to downsize is taken by majority approval, that is, $k=n / 2+1$ if $n$ is even, and $k=(n+1) / 2$ if $n$ is odd. To simplify matters, take a step function $\tau^{n}(\cdot)$ such that $\tau^{n}(a)=0$ if $a<n-\kappa$, and $\tau^{n}(a)=\tau$ otherwise. ${ }^{19}$ Table 1 computes the optimal scapegoat size of Proposition 1 for different values of $n, \kappa$ and $\mu$.

A few things are interesting to note. First, the optimal scapegoat size is weakly increasing in the probability $\mu$. That is, the higher the likely number of pathological individualist, the (weakly) bigger the size of the expelled group. Second, the values for the optimal scapegoat size range from 0 to $n / 2-1$, which is the biggest size that can be expelled by majority approval. ${ }^{20}$ The cells with zeros correspond to $\mu n<\kappa$, when the expected number of pathological individualists is lower than the provision point $\kappa$. When $\mu n \geq \kappa$, absent downsizing and given that pathological individualists always choose not to contribute, individual returns would always be zero irrespective of the choices of $a_{i}$. Given such bad prospects, downsizing is collectively approved and the optimal scapegoat size is $0<c^{*} \leq n / 2-1$. The upper bound $n / 2-1$ is obtained with bad enough prospects, that is, with high enough values for $\mu .^{21,22}$

19. The actual value of $\tau$ is left unspecified and is fixed for each value of $n, \kappa, \mu$ so that the equilibrium existence in Proposition 1 conditions hold true for some $c$.

20. More precisely, the highest scapegoat size than can be expelled is equal to $n / 2-1$ when the population size $n$ is even, as in the numerical examples of Table 1 . If instead $n$ is odd, the highest possible scapegoat size is equal to $(n-1) / 2$.

21. Formally, denote by $[(n-1) / 2]$ the highest integer smaller or equal than $(n-1) / 2$. Majority rule requires that the optimal size $c^{*}$ maximizes $h(\mu, n-c)$ on $\{0,1, \ldots,[(n-1) / 2]\}$. Consistent with Proposition 3, (see Section 6.2) $c^{*}=0$ when $\mu n<\kappa$. When $\mu n \geq \kappa, c^{*}$ is an "interior" point (i.e., $\left.1 \leq c^{*}<[(n-1) / 2]\right)$ for low values of $\mu$, and $c^{*}=[(n-1) / 2]$ when $\mu$ is high enough.

22. When the optimal scapegoat size is equal to $n-k$, this "optimum" is constrained by the $k$ majority approval rule. This could lead to a temptation to repeat the downsizing, and may even unravel the downsizing process. Without the possibility of commitment, one could assume that only the members not included in $C$ could vote on $C$ exclusion. 


\subsection{Smooth Payoff Functions: An Analytic Example}

Assume $\tau^{n}(k)=\tau \beta^{k-n}$, where $\beta, \tau>1$. Thus, $\tau^{n}(k)$ is an increasing and convex function of the number $k$ of contributors. ${ }^{23}$

Using (1), the expected return from the cooperative action when everybody plans to contribute are

$$
H(n)=\tau\left(\frac{\mu}{\beta}+1-\mu\right)^{n-1} F(n)=\tau \alpha^{n-1} n,
$$

where $\alpha=\mu / \beta+(1-\mu)<1$ for all $0<\mu<1$.

We discuss the comparative statics on the optimal size of the expelled group.

Let $c$ be the size of the expelled group. The equilibrium payoff in Proposition 1 is now $G(n-c)=(1-\mu) \tau \alpha^{n-c-1}(n-c)+\mu(n-c)$, and the optimal size of the expelled group solves

$$
n-c^{*} \in \arg \max \{G(n-c): n-k \leq n-c \leq n\} .
$$

It is fairly obvious that the optimum $c^{*}$ sometimes coincides with $k$ (as we saw in the previous example). We focus instead on the comparative statics of interior solutions.

At an interior optimum we have $G^{\prime}\left(n-c^{*}\right)=0$ and $G^{\prime \prime}\left(n-c^{*}\right) \leq 0$. As usual, we determine the comparative statics of $c^{*}$ with respect to the exogenous payoff parameters by differentiating the first-order optimality condition with respect to $\tau, \beta$ and $\mu$, and by using the implicit function theorem.

We first analyze the effect of the level $\tau$ of the returns to cooperation. We obtain

$$
\frac{\partial c^{*}}{\partial \tau}=-\frac{1}{G^{\prime \prime}\left(n-c^{*}\right)} \frac{\mu}{\tau} \geq 0,
$$

that is, $c^{*}$ and $\tau$ move in the same direction. This is because a higher $\tau$ increases the benefits of coordination, and thus the losses incurred by having PIs in the group.

We now analyze the impact of $\beta$, the marginal returns to cooperation. Routine algebra leads to $\partial c^{*} / \partial \beta \geq 0$ if and only if

$$
\tau \alpha^{n-c^{*}-1}>\frac{n-c^{*}-1}{n-c^{*}} \frac{\mu}{1-\mu} .
$$

With $n$ large, this condition boils down to the coordination multiplier in the downsized organization, $\tau \alpha^{n-c^{*}-1}$, being higher than the odds of being a PI,

23. Notice that $\tau^{m}(m-k)=\tau \beta^{-k}=\tau^{n}(n-k)$, for all $m>n$, so that all the assumptions on the returns to coordination are satisfied. 
$\mu /(1-\mu)$. Thus, everything else equal, a high value of $\tau$ or a low value of $\mu$ make it more likely that the optimal size of the expelled group increases with $\beta$. This is because a higher $\beta$ increases the benefits of coordination and thus the losses incurred by having PIs in the group. ${ }^{24}$

Finally, we analyze the impact of the probability $\mu$ to become a PI on the optimal size of the expelled group. Again, when $n$ is large, $\partial c^{*} / \partial \mu \geq 0$ if the odds of being a $P I$ are low enough compared to the returns to cooperation, for example, when $\tau \alpha^{n-c^{*}-1}>\mu /(1-\mu)$.

It is clear from the previous calculations that the value of $\mu$ is important in determining the sign of the impact of $\beta$ and $\mu$ on the optimal size of the expelled group, as well as the level of the impact of $\tau$ on this size. There is a simple heuristic argument that makes this clear.

The expected payoff from downsizing is $(1-\mu) H(n-c)+\mu F(n-c)$. This is the weighted sums of equilibrium payoffs for the $P I$ and for the non-PI individual.

For the $P I$, who does not care about cooperation and obtains $F(n-c)$ in the downsized organization, the optimal value for $c$ is zero.

For the non-PI, who does care about cooperation, the optimal size of the expelled group maximizes $H(n-c)$. At an interior optimum, this optimal size is $-1 / \ln a$, an increasing function of $\mu$ and $\beta$.

The overall optimal value of $c$ solves the tension between the two optimal values for the $P I$ and for the non- $P I$, and how this tension is actually solved depends on the relative weight of being a $P I$ versus a non- $P I$ type, given by the odds $\mu /(1-\mu)$. When these odds are small, the optimal size of the expelled group inherits the properties from the non-PI optimum, and the effect of $\mu$ and $\beta$ are positive. How big or small this ratio needs to be is determined by the relative payoffs accruing to both types, which is $\tau a^{n-c^{*}-1}$.

\section{Endogenous Expelled Group}

Let's now endogenize the choice of the set $C$. Prior to the approval or rejection of $C$, the members of the organization must choose a person who will propose the set to be subject to the approval/rejection process.

COROLLARY 1. Consider any collective choice procedure that selects a delegate to make the proposal of $\mathrm{C}$, and suppose that $\mathrm{k}+\mathrm{c} \leq \mathrm{n}$. Then the delegate proposes a candidate $\mathrm{C}$ which maximizes $\mathrm{H}(\mathrm{n}-\mathrm{c})$. In all strategy profiles that survive two

24. This is similar to the effect of $\tau$, except that there is now a counteravailing effect, because increasing $\beta$ makes more costly a given decrease in the size of the expelled group (notice that $\beta$ affects both the level and the slope of the reduction costs). 
rounds of deletion of weakly dominated strategies, all players $\mathrm{i} \in \mathrm{N} \backslash\{\mathrm{C} \cup \mathrm{PI}\}$ vote for elimination of $\mathrm{C}$ and choose $\mathrm{a}_{\mathrm{i}}=1$ after the elimination.

Proof. Once a delegate is chosen, Proposition 1 characterizes the behavior she expects for all proposals. Therefore, the delegate will propose a set $C$ (not including herself) which maximizes $H(n-c)$, thus her expected payoff $(1-\mu) H(n-c)+\mu F(n){ }^{25}$

Downsizing does effectively rebuild team spirit even when the scapegoat is endogenously selected. The selection, here, consists of a two-stage procedure whereby members first choose a representative who then proposes a candidate subgroup for elimination to their approval. Note that the collective choice procedure for the selection of the representative is left unspecified, implying that the result holds with a huge variety of institutional arrangements.

\section{The Case of Unmediated Downsizing}

We now consider the general case where the scapegoat is endogenously and directly selected by organization members, without resorting to representatives. We contemplate a voting procedure where members do not simply cast a yes/no vote between two given alternatives. Rather, they vote over the precise identity of each member to be expelled. This potentially opens the door to strategic behavior during the voting stage.

\subsection{Coordination Problems in Unmediated Downsizing}

The corporate downsizing procedure involves all initial members of the organization. Each member submits a list of individuals to be expelled from the organization. We denote by $N$ the set of initial members of the organization and by $S_{i} \subseteq N$ the list submitted by member $i$. The case $S_{i}=\emptyset$ corresponds to $i$ submitting a list with no names.

Given a collection of submitted lists $\mathcal{S}=\left(S_{1}, \ldots, S_{n}\right)$, a collective choice procedure determines the group $E(\mathcal{S})$ that is actually expelled. $E(\mathcal{S})$ is selected the following way. If there is a unique group $S^{\prime}$ that receives the (absolute) majority of votes, then $E(\mathcal{S})=S^{\prime}$. In all other cases, $E(\mathcal{S})=\emptyset$. We denote by $e(\mathcal{S})$ the cardinality of $E(\mathcal{S})$.

25. Note that the delegate is not in $P I$ when choosing the candidate subgroup to elimination as types are defined at the interim stage between the voting stage and the contribution stage, that is, after the choice of $C$ by the delegate. 
PROPOSITION 2. Suppose $\mathrm{H}(\mathrm{n}-\mathrm{e}(\mathcal{S}))>\mathrm{F}(\mathrm{n})>\mathrm{H}(\mathrm{n})$. In all strategy profiles that survive two rounds of deletion of weakly dominated strategies where $\mathrm{E}(\mathcal{S}) \neq$ $\emptyset$, all players $\mathrm{i} \notin \mathrm{PI}$ such that $\mathrm{S}_{\mathrm{i}}=\mathrm{E}(\mathcal{S})$ choose $\mathrm{a}_{\mathrm{i}}=1$.

Proof. We first show that $F(n)>H(n)$ implies that, for all $i \notin P I$, submitting a list $S_{i} \neq \emptyset$ and not contributing whenever $S_{i}$ is eliminated is a weakly dominated strategy. Consider the following strategy for player $i$ : submit a list $S_{i} \neq \emptyset$, then after some history where $S_{i}$ is eliminated, $a_{i}=0$ (we do not need to specify the full strategy). Consider an alternative strategy that behaves as the previous one after all histories, expect that it submits $S_{i}=\emptyset$. We show that the latter dominates the former.

First note that the expected payoff of a player when nobody is eliminated and she chooses $a_{i}=0$ is $F(n)$. The payoff for player $i \in N \backslash\{E(\mathcal{S}) \cup P I\}$ when $E(\mathcal{S}) \neq \emptyset$ is eliminated and she chooses $a_{i}=0$ is $F(n-e(\mathcal{S}))$, and $F(n-e(\mathcal{S}))<F(n)$. Note also that $F(n)>H(n)$ implies that it is dominant to choose $a_{i}=0$ when nobody is eliminated.

Consider now some list of submissions $S_{-i}$. We distinguish two cases. First, player $i$ 's choice is pivotal. If she votes $S_{i} \neq \emptyset$ then $E\left(S_{i}, S_{-i}\right) \in\left\{S_{i}, \emptyset\right\}$, whereas if she votes $S_{i}=\emptyset$ then $E\left(\emptyset, S_{-i}\right)=\emptyset$. Thus, the payoff accruing to $i$ is never lower under the second strategy and is strictly higher when $E\left(S_{i}, S_{-i}\right)=S_{i}$. The alternative case is trivial. The choice of $i$ is not pivotal, and behavior (thus payoffs) after all histories is the same for both strategies.

Once agents know that every non- $P I$ individual will contribute when their submitted list of players is eliminated, the result follows from the fact that $H(n-$ $e(\mathcal{S}))>F(n)$ implies that payoffs are higher with and without elimination.

This shows that approving to downsize the organization is a possibility, but, unlike in the centralized case, it is not the only reasonable possibility. ${ }^{26}$ Indeed, the lack of a centralized proposer adds a different coordination problem to be collectively solved, namely, identifying the group to be expelled. But because the pressure to switch from a bad equilibrium to a good one is large, a focal point could be used to choose a group to be eliminated. As we already discussed, Girard (1982) argues that scapegoats have a mark, which distinguishes them for the rest of society. This could serve as the focal point. However, a focal point may not solve the problem optimally, because the group identified for elimination may be larger or smaller than the one maximizing $H(n-c)$. In reality, firms typically have a policy for layoffs, most usually the last hired are the first to be laid off. Another intriguing possibility, which we explore in the following subsection more formally is that history can serve to efficiently focus expectations.

26. In fact, it is an iteratively undominated equilibrium outcome both to have no downsizing as well as "suboptimal" downsizing. 


\subsection{Learning Dynamics or an Algorithm for Efficiency}

The preceding discussion leaves open the possibility that a fully decentralized downsizing procedure may lead to suboptimal equilibrium allocations. One could think that this scarcely matters, because we have shown in Proposition 1 that a choice through a delegate already reaches the maximum organizational output. There may be circumstances, however, where the direct route would be better. We have not considered, for example, the case where delegates are corruptiblewhich could get in the way of the delicate belief-coordinating process.

Given these possibilities, is there any way to justify a fully decentralized downsizing? Suppose all agents are sufficiently strategically savvy to behave as in Proposition 2, but they have more trouble guessing at the particular way others will vote, and have to learn their way through equilibrium by a trial-and-error process. More specifically, we assume that agents play the game repeatedly. Each individual starts by playing some arbitrary (pure) voting-strategy (as we said before, they still behave as in Proposition 2 after the voting stage). Before each repetition of the game, they have an opportunity to change their vote with some probability. The dynamics of voting will be fully described when one identifies the transition probabilities between strategies. Instead of fully describing the process, we enumerate a set of assumptions that are sufficient for the results of the paper.

D1. The transition probabilities depend exclusively on the present voting profile.

D2. One individual, chosen at random, is given the chance to update her vote every period.

D3. If the individual is given the chance to update her strategy, and she does not already enjoy the maximal possible payoff in the game, any vote that best responds to the present voting profile is adopted with positive probability.

D4. If the individual already enjoys the maximal possible payoff in the game, she changes her vote with zero probability.

These assumptions permit us to obtain clear-cut results in a relatively simple fashion. Assumption D1 simplifies the analysis by making the strategy profile of a certain period the state variable of the system, but it is not essential for the results. It would suffice if the system had a finite memory, for example. Assumption D2 is necessary because the voting game is such that except when voters are pivotal, any vote is a best response. The fact that every vote is a best response, coupled with assumption D3, would make it impossible to find any stable outcome, as all voters could simultaneously switch to anything else and destroy the stability of any outcome.

Assumptions D3 and D4 are designed to exploit a special characteristic of the voting game. While an agent is not pivotal, she can change her vote while choosing a best response. This easily leads to a situation in which someone is pivotal. At that point only votes that are "efficient" are taken. 
Properties D1 to D4 make our dynamics similar to the ones in Kim and Sobel (1995). Assumption D2 corresponds to their assumption (I), Assumption D3 corresponds to their assumption (BR), assumption D4 to their assumption (NL). Our dynamics are also closely related to the ones in Hurkens (1995) and Gilboa and Matsui (1991).

Proposition 3. Let $\mathcal{S}^{\mathrm{C}}$ be any set of voting profiles which leads to a set of eliminated individuals $\mathrm{C}$ that maximizes $\mathrm{H}(\mathrm{n}-\mathrm{c})$, let $\mathcal{C}$ be the union of all such sets, and let $\mathcal{S}^{\mathcal{C}}=\cup_{\mathrm{C} \in \mathcal{C}} \mathcal{S}^{\mathrm{C}}$. Given dynamics that satisfy properties D1, D2, D3, D4:

(a) If $\mathcal{S}(0)$ is such that $\mathcal{S}(0) \notin \mathcal{S}^{\mathrm{C}}$, then, for all $\mathrm{C} \in \mathcal{C}, \operatorname{Pr}\left(\right.$ for some $\mathrm{t}^{\prime}: \mathcal{S}(\mathrm{t}) \in$ $\left.\mathcal{S}^{\mathrm{C}} \forall \mathrm{t} \geq \mathrm{t}^{\prime}\right)>0$.

(b) $\operatorname{Pr}\left(\right.$ for some $\left.\mathrm{t}^{\prime}: \mathcal{S}(\mathrm{t}) \in \mathcal{S}^{\mathcal{C}} \forall \mathrm{t} \geq \mathrm{t}^{\prime}\right)=1$.

Proof. To prove (a) we have to look at a number of cases:

Case 1. Suppose that $\mathcal{S}(0)$ is such that nobody is eliminated and nobody can change the majority. Note that all votes are a best response because none changes the outcome, and because nobody is eliminated, no player obtains the highest payoff. Then, by assumptions $\mathrm{D} 2$ and D3, there is positive probability of a sequence of one period moves where all members of the population sequentially change their vote to $S_{i}=\emptyset$ so that we end up in a state of the population where $S_{i}(t)=\emptyset$, for all $\mathrm{i}$. Then, from that state and for any $C^{*} \in \mathcal{C}$, there is, by assumptions D2 and D3, a positive probability that all members of $N \backslash C^{*}$ get sequentially a chance to vote and they choose $S_{i}=C^{*}$, which is a bestresponse even when this means a change in state from not eliminating anybody to eliminating $C^{*}$. Once $\mathcal{S}(t)$ is such that $C^{*} \in \mathcal{C}$ is eliminated, no member of $N \backslash C^{*}$ changes her vote, by $\mathrm{D} 4$.

Case 2. Suppose that $\mathcal{S}(0)$ is such that set $C^{*} \notin \mathcal{C}$ is eliminated. Then by assumptions D2 and D3, there is a positive probability of a sequence of one period moves where all members of the population sequentially change their vote to $S_{i}=C^{*}$ so that we end up in a state of the population where $S_{i}(t)=C^{*}$, for all $i$. From that point, there is a positive probability of a sequence of one period moves where all members of the population sequentially change their vote to $S_{i}=\emptyset$ so that we end up in a state of the population where $S_{i}(t)=\emptyset$, for all $i$. The only thing needed for this to work is that the person in the sequence who changes from a majority voting to eliminate $C^{*}$ to a $\emptyset$-eliminating majority is an agent $i \in C^{*}$. Once in state $S_{i}(t)=\emptyset$, for all $i$ we can apply the same reasoning as in Case 1.

Case 3. Suppose that $\mathcal{S}(0)$ is such that nobody is eliminated and some agents can change the majority to eliminating set $C^{*}$. Then by assumption D2, one of the agents $i$ already voting for $C^{*}$ gets to move and changes her vote to $S_{i}=\emptyset$. From that point on we are in a situation like Case 1. 
Case 4. Suppose that $\mathcal{S}(0)$ is such that set $C^{*} \in \mathcal{C}$ is eliminated, and that the number of players voting in favor of the elimination of $C^{*}$ which belong to $N \backslash C^{*}$ is $l \geq k$. Then none of those players will ever change their votes and $S_{i}(t)=C^{*}$ for all $t$.

Case 5. Suppose that $\mathcal{S}(0)$ is such that set $C^{*} \in \mathcal{C}$ is eliminated, and that the number of players voting in favor of the elimination of $C^{*}$ which belong to $N \backslash C^{*}$ is $l<k$. Then by assumptions D2 and D3, there is a positive probability of a sequence of one period moves where $l-k$ members of $N \backslash C^{*}$ not voting already for $C^{*}$ change their vote to $S_{i}=C^{*}$. From that point on, we can apply the same reasoning as in Case 4, and the state will always be $S_{i}(t)=C^{*}$ after that time.

Cases 1 through 5 exhaust all the possible cases to show part (a), so (a) follows. To establish part (b), notice that part (a) establishes that from any $\mathcal{S}(t)$ there is a lower bound $\varepsilon>0$ on the probability of reaching $\mathcal{S}^{\mathcal{C}}$, and staying there forever in a number of steps smaller than some fixed and finite $\eta$. So the probability of not reaching $\mathcal{S}^{\mathcal{C}}$ in $\eta n$ steps is bounded above by $(1-\varepsilon)^{\eta n}$. Because $\lim _{n \rightarrow \infty}(1-\varepsilon)^{\eta n}=0$, part (b) follows.

\section{A Different Timing for Type Revelation}

The timing of type revelation may be seen as somewhat awkward in the game we just presented. One could perhaps expect that individuals knew whether they were $P I$ at the time of voting, and not just when choosing whether to cooperate. It is not completely clear, however, that this is clearly the best assumption, as there is substantial evidence (Johansson-Stenman and Svedsäter 2003) and theorizing (Santos-Pinto and Sobel 2005) about the importance of self-image for economic agents, and voting as a PI could negatively affect the individual's self-image in a way that actually acting as a $P I$ would not. Nevertheless, we will now see that the results are very similar when one uses the alternative assumption that individuals know their type at the time of participating in the collective decision scheme.

We assume that collective choice procedures are anonymous, in the sense that voting or related activities will be secret. So agents will only know how many others are in favor or against different options, not who is it that favors them. Besides being realistic, this trait would help true revelation of information. If voting were not secret, a PI-type would be concerned that signalling his type through voting would expose her to exclusion from the organization.

In addition, we assume that the participants will only be informed about whether the proposal was passed or not. This is not quite as realistic, but the alternative provides the opportunity for much more conditioning of actions by the workers on the results of voting, and hence the possibility for strange equilibria to arise. We feel that those equilibria are probably unreasonable, but cannot give a more formal rebuttal, whereas under the assumption we use, the results are sharper, 
and it is still a feasible mechanism, which gives it, at a minimum, normative relevance.

The belief that other individuals are $P I$ is now dependent on the history of play. Let $\mathcal{N}$ be the outcome of voting under which $C$ is not eliminated. Denote by $\pi$ the cardinality of $P I$, by $p(\cdot \mid \mathcal{N})$ the posterior distribution over the size of $P I$ and by $p_{0}(\cdot)$ the prior distribution over $\pi$, which depends on $\mu$.

With this we can now proceed to review our results.

PROPOSITION 4. Assume that $\tau^{\mathrm{n}-\mathrm{c}}(\mathrm{k}) \mathrm{F}(\mathrm{n}-\mathrm{c})>\mathrm{F}(\mathrm{n})>\mathrm{H}(\mathrm{n})$. In all strategy profiles that survive two rounds of deletion of weakly dominated strategies, all players $\mathrm{i} \in \mathrm{N} \backslash\{\mathrm{C} \cup \mathrm{PI}\}$ vote for elimination of $\mathrm{C}$ and choose $\mathrm{a}_{\mathrm{i}}=1$ after the elimination.

Proof. Notice that for PI types it is always dominant to reject an elimination. Indeed, their payoff after elimination is $F(n-c)$, which is smaller than $F(n)$, the payoff without elimination, and their vote may be pivotal.

We will now show that non- $P I$ types choose to eliminate and then cooperate when $\tau^{n-c}(k) F(n-c)>F(n)>H(n)$. First, voting to eliminate and then choosing $a_{i}=0$ is dominated for the reasons we have mentioned in other cases. Therefore, we are left to show that voting not to eliminate cannot be optimal when $\tau^{n-c}(k) F(n-c)>F(n)>H(n)$. We proceed in a number of steps.

Step 1. After observing an elimination, the distribution of the number of $P I$-types is a first-order stochastically dominating shift over the prior distribution.

Proof of Step 1. Denote by $\rho_{S}$ the cardinality of the number of non-PI players who decide not to cooperate after observing the outcome $\mathcal{N}$ under strategy profile $S$. Given such strategy profile $S$, let $P$ be the corresponding probability that the outcome is $\mathcal{N}$. Let $m=n-k$ and $p_{0}^{i}=p_{0}(\pi=i)$. For $x \leq m$, we have that

$$
\begin{aligned}
& p(\pi \leq x \mid \mathcal{N}) \\
& =\frac{\sum_{i=0}^{x} p_{0}^{i} P\left(\rho_{S}+i \geq m+1\right)}{\sum_{i=0}^{x} p_{0}^{i} P\left(\rho_{S}+i \geq m+1\right)+\sum_{i=x+1}^{n-k} p_{0}^{i} P\left(\rho_{S}+i \geq m+1\right)+\sum_{n-k+1}^{n} p_{0}^{i}} \\
& \leq \frac{\sum_{i=0}^{x} p_{0}^{i} P\left(\rho_{S}+x \geq m+1\right)}{\sum_{i=0}^{x} p_{0}^{i} P\left(\rho_{S}+x \geq m+1\right)+\sum_{i=x+1}^{n-k} p_{0}^{i} P\left(\rho_{S}+x \geq m+1\right)+\sum_{n-k+1}^{n} p_{0}^{i}} \\
& \leq \frac{\sum_{i=0}^{x} p_{0}^{i}}{\sum_{i=0}^{x} p_{0}^{i}+\sum_{i=x+1}^{n-k} p_{0}^{i}+\left(\sum_{n-k+1}^{n} p_{0}^{i}\right) / P\left(\rho_{S}+i \geq m+1\right)} \\
& \leq \frac{\sum_{i=0}^{x} p_{0}^{i}}{\sum_{i=0}^{x} p_{0}^{i}+\sum_{i=x+1}^{n-k} p_{0}^{i}+\sum_{n-k+1}^{n} p_{0}^{i}} \\
& =\sum_{i=0}^{x} p_{0}^{i}=p_{0}(\pi \leq x),
\end{aligned}
$$


where the first inequality follows because the function $x_{1} /\left(x_{1}+x_{2}+x_{3}\right)$ is increasing in $x_{1}$, and decreasing in $x_{2}$, and we have substituted $P\left(\rho_{S}+i \geq m+1\right)$ in the positions corresponding to $x_{1}$ and $x_{2}$ by something respectively bigger and smaller.

Step 2. The payoff for a non-PI-type for cooperating after observing the rejection of an elimination is bounded above by $H(n)$.

Proof of Step 2. Given that PI-types do not cooperate, the best possible case for cooperation is when all non-PI types cooperate. Thus the payoff for cooperation is bounded by $\sum_{i=0}^{n} p(\pi=i \mid \mathcal{N}) \tau^{n}(n-i) F(n)$. We know by Step 1 that $p(\pi=i \mid \mathcal{N})$ is a first-order stochastically dominating shift over $p_{0}(\pi=i)$. Because $\tau(n-i)$ is a monotonic function, this implies (Mas-Colell, Whinston, and Green 1995, definition and proposition 6.D.1), that the payoff for cooperation is bounded by $\sum_{i=0}^{n} p_{0}(\pi=i) \tau^{n}(n-i) F(n)=H(n)$.

Step 3. When $F(n)>H(n)$, cooperating for a non-PI after observing $\mathcal{N}$ is not optimal.

Proof of Step 3. $F(n)$ is the payoff for cooperating after $\mathcal{N}$, and $H(n)$ is an upper bound to the payoff under cooperation, by Step 2 .

Step 4. When $\tau^{n-c}(k) F(n-c)>F(n)$, voting for the elimination of $C$ (and then choosing $a_{i}=1$ after elimination) is weakly dominant if all players avoid the use of weakly dominated strategies.

Proof of Step 4. Indeed, when $C$ is eliminated, at least $k$ players cast the yes vote. Then, $\tau^{n-c}(k) F(n-c)$ is a lower bound for players in $C \cup P I$ that contribute after $C$ is eliminated.

With this we can now proceed to the following result, analogous to Proposition 1.

COROLLARY 2. Consider any collective choice procedure that selects a delegate to make the proposal of $\mathrm{C}$ and suppose that $\mathrm{k}+\mathrm{c} \leq \mathrm{n}$. Then, if the delegate is not of a $\mathrm{PI}-t y p e$, she proposes a candidate $\mathrm{C}$ which maximizes the ex ante payoff of all $\mathrm{i} \in \mathrm{N} \backslash\{\mathrm{C} \cup \mathrm{PI}\}$.

\section{The Optimal Size of the Organization}

We have uncovered a mechanism that supports optimal downward reorganizations. Could something similar work in the upward direction? If so, we would be closer to having a complete theory of firm size in our context. At the risk of outstretching our arguments, we can say that under certain technological conditions this may be indeed so.

Suppose that there are two organization sizes $n^{*}>n$ such that $\tau^{n^{*}}\left(n^{*}\right)$ $F\left(n^{*}\right)>\tau^{n}(n) F(n)$. So the size $n^{*}$ is more efficient than $n$. The initial size is $n$, and the organization would like to increase its size to $n^{*}$. Now assume 
that $\tau^{n}(n) F(n)>F\left(n^{*}\right)$. In words, the cooperative outcome in the smaller organization is more profitable than the noncooperative outcome in the larger organization. Notice that this assumption is weaker than that required in Proposition 1 for successful downsizing from $n^{*}$ to $n$, so the same (stronger) assumption can be used for both downsizing and upsizing. In addition we will require that $\tau^{n^{*}}(k) F\left(n^{*}\right)>\tau^{n}(n) F(n)$, that is, only $k$ cooperators (in general smaller than $n^{*}$ ), are needed for cooperation to be profitable in the larger organization. ${ }^{27}$ Suppose, in addition that there are no PI-types in this environment (so $\mu=0$ ).

As in the case of corporate downsizing, upsizing requires $k$-majority approval. Now we will show that if it is common knowledge that all members of the initial organization cooperate, a $k$-majority vote on the hiring of $n^{*}-n$ new members for the organization will lead to an organization of the increased, more efficient, size, where everybody will cooperate.

PROPOSITION 5. Suppose that initially we have an organization $\mathrm{N}$ of size $\mathrm{n}$, where $\mathrm{a}_{\mathrm{i}}=1$, for all $\mathrm{i} \in \mathrm{N}$. If $\tau^{\mathrm{n}^{*}}(\mathrm{k}) \mathrm{F}\left(\mathrm{n}^{*}\right)>\tau^{\mathrm{n}}(\mathrm{n}) \mathrm{F}(\mathrm{n})>\mathrm{F}\left(\mathrm{n}^{*}\right)$ then, in all strategy profiles that survive two rounds of deletion of weakly dominated strategies, all players $\mathrm{i} \in \mathrm{N}$ vote for hiring the $\mathrm{n}^{*}-\mathrm{n}$ new members, and all members of the enlarged organization $\mathrm{N}^{*}$ choose to contribute, that is, $\mathrm{a}_{\mathrm{i}}=1$, for all $\mathrm{i} \in \mathrm{N}^{*}$.

Proof. First, observe that for all $i \in N$ approving the enlargement and then choosing $a_{i}=0$ is weakly dominated by not approving the enlargement and then choosing $a_{i}=1$. The payoff without enlargement is $\tau^{n}(n) F(n)$ by the assumption that at the initial size $n$, all individuals choose $a_{i}=1$. The payoff after enlargement when choosing $a_{i}=0$ is $F\left(n^{*}\right)$. Because casting a vote for the enlargement may be pivotal for this enlargement, and $\tau^{n}(n) F(n)>F\left(n^{*}\right)$, the domination follows. Thus, any player who votes for enlargement will play $a_{i}=1$. Therefore a lower bound for the expected payoff for any $i$ for the choice of $a_{i}=1$ in case of enlargement is given by $\tau^{n^{*}}(k) F\left(n^{*}\right)$. The condition $\tau^{n^{*}}(k) F\left(n^{*}\right)>\tau^{n}(n) F(n)$ guarantees, then, that the player $i \in N$ prefers the situation where the organization is enlarged. Because casting a vote for the enlargement by any player $i$ may be pivotal for this enlargement, it is dominant to vote for this elimination (and then choose $a_{i}=1$ ).

Where does this leave us? It is not too difficult to see that by iterating the argument in the proposition above there are technological conditions under which repeated consensual enlargements (starting with a size of 1 , when cooperation is guaranteed) could lead to an organization of optimal size where all members cooperated. This organization could be buffeted by shocks leading to the loss

27. Given earlier assumptions, this will happen provided the increase in organization size from $n$ to $n^{*}$ is not too large, and the majority required for enlargement $k$ is sufficiently large. 
of cooperation (the appearance of $P I$-types). Downsizing could then lead to a recuperation of the culture of cooperation. After that, the organization could regain once more its optimal size by consensual enlargement.

\section{Discussion and Conclusion}

We have shown in this paper that downsizing can have efficiency-enhancing effects in organizations, by focusing expectations on the cooperating outcome. Our results rely on two basic ingredients. One is the view that firms exist to generate synergies and solve the coordination problems these synergies induce. The other important element is the fact that collective choices can "signal" intended plans of action. One could perhaps question the necessity of such a cumbersome and centralized mechanism as "voting" about downsizing in a modern organization. It would seem, perhaps, as effective to merely have players make a promise to choose the high action. We have two answers for this question. First, the "voting" can be merely an informal "consultation," decentralized to divisional managers. ${ }^{28}$ All that is important is that the outcome of this process is common knowledge and believed to be nonmanipulated. Second, the alternative "written promise" preserves the bad equilibrium if it is not enforceable. And it is not enforceable, if it is not verifiable, which seems to us the natural application of our setting. Think of the organization as a bank that provides a variety of services to a business customer. The customer is only satisfied if the loan officer, the risk analyst, the account manager, and the mergers advisor all provide timely and friendly service. But if he is dissatisfied and goes to the competition, it may be very difficult to know who is really guilty. The customer may not tell the bank's division manager of the reason for his decision, or he may not really know (the account manager may have been late with his proposal because the risk analyst did not cooperate with him in his efforts).

Seminal papers on coordination in firms are Kreps (1990) and Milgrom and Roberts (1995). Downsizing in our model has the property that it induces people to believe that others will play the high payoff (but risky) action. In this sense it acts in the way that Kreps envisioned (good) corporate culture (see also Crémer (1993) and Lazear (1995). Our mechanism, though, is different from the one in that paper, which relied mostly on experiences shared by organization members to coordinate beliefs on the right action. Here, by contrast, a shared experience of cheating induces people to believe that a large enough number of pathological individualists are present in the organization. Only the downsizing event will change those beliefs for the good.

28. In fact, formal consultation of downsizing with workers (often in workers' councils) is mandatory in most European countries, as we discussed in the Introduction. 
Demange (2004) has also studied how organizations choose their structure as a way to induce coordination. Her approach is quite different to ours. She has studied the problems created in organizations which need to coordinate decisions of the whole group, in a context where subgroups (coalitions) are assigned decision powers beyond those of individuals. In that context she has shown that hierarchical organizations induce the highest possible level of stability, at the time that efficiency is preserved. ${ }^{29}$

The relationship between scapegoats and organizations has also been studied by Winter (2004). He studies the incentive effects in a team-production problem carried out by a hierarchy of selecting certain individuals for punishment in case of an organizational failure. The mechanism design problem consists of finding the best possible structure of punishments for a given organization.

\section{References}

Amabile, Teresa M., and Regina Conti (1999). "Changes in the Work Environment for Creativity During Downsizing." Academy of Management Journal, 42, 630-641.

Baily, Martin Neil, Eric J. Bartelsman, and John Haltiwanger (1996). "Downsizing and Productivity Growth: Myth or Reality?" In Sources of Productivity Growth in the 1980's, edited by D. G. Mayes. Cambridge University Press.

Barnes, Matthew, and Jonathan Haskel (2001). "Productivity, Competition and Downsizing." Working paper, Queen Mary College.

Bassi, Laurie J., and Mark E. Van Buren (1997). "Sustaining High Performance in Bad Times." Training and Development, 51, 32-42.

Ben-Porath, Elchanan, and Eddie Dekel (1992). "Signaling Future Actions and the Potential for Sacrifice." Journal of Economic Theory, 57, 36-51.

Bolton, Patrick, and Matthias Dewatripont (1994). "The Firm as a Communication Network." Quarterly Journal of Economics, 109, 809-839.

Borgen, William A. (2000). "Developing Partnerships to Meet Clients' Needs in Changing Government Organizations: A Consultative Process." Journal of Employment Counseling, $37,128-143$.

Brandts, Jordi, Antonio Cabrales, and Gary Charness (2006). "Forward Induction and the Excess Capacity Puzzle: An Experimental Investigation.” Economic Theory.

Broseta, Bruno, Enrique Fatás, and Tibor Neugebauer (2003). "Asset Markets and Equilibrium Selection in Public Goods Games with Provision Points: An Experimental Study." Economic Inquiry, 41, 574-591.

Chadwick, Clint, Larry W. Hunter, Stephen L. Walston (2004). "Effects of Downsizing Practices on the Performance of Hospitals." Strategic Management Journal, 25, 405-427.

Crémer, Jacques (1993). "Corporate Culture: Cognitive Aspects." Industrial and Corporate Change, 3, 351-386.

Demange, Gabrielle (2004). "On Group Stability in Hierarchies and Networks." Journal of Political Economy, 112, 754-778.

29. This research is, in turn, connected with the large literature exploring communication and network formation in organizations, one of whose most important aims is precisely to explain the ubiquity of hierarchies in organizations. See, for example, Radner (1993), Bolton and Dewatripont (1994), van Zandt (1999), Garicano (2000), or Guimerà et al. (2002). 
Foster, Lucia, John Haltiwanger, and C. Krizan (2001). "Aggregate Productivity Growth: Lessons from Microeconomic Evidence." In New Directions in Productivity Analysis, edited by Edward Dean, Michael Harper, and Charles Hulten. University of Chicago Press.

Garicano, Luis (2000). "Hierarchies and the Organization of Knowledge in Production." Journal of Political Economy, 108, 874-904.

Gilboa, Itzhak, and Akihiko Matsui (1991). "Social Stability and Equilibrium." Econometrica, $59,859-867$.

Girard, René (1982). The Scapegoat (translated from French by Y. Freccero). Johns Hopkins University Press (1986).

Guimerà, Roger, Albert Díaz-Guilera, Fermando Vega-Redondo, Antonio Cabrales, and Àlex Arenas (2002), "Optical Network Topologies for Local Search with Congeostion." Physical Review Letters, 89, 248701.

Heywood, John S., and Uwe Jirjahn (2004). "Teams, Teamwork and Absence.” Scandinavian Journal of Economics, 106, 765-782.

Hurkens, Sjaak (1995). "Learning by Forgetful Players." Games and Economic Behavior, 11, 304-329.

Johansson-Stenman, Olof, and Henrik Svedsäter (2003). "Self Image And Choice Experiments: Hypothetical And Actual Willingness To Pay.” Working paper, Göteborg University.

Kim, Yong-Gwan, and Joel Sobel (1995). "An Evolutionary Approach to Pre-Play Communication." Econometrica, 63, 1181-1193.

Kremer, Michael (1993). "The O-Ring Theory of Economic Development." Quarterly Journal of Economics, 108, 551-575.

Kreps, David M. (1990). "Corporate Culture and Economic Theory.” In Perspectives on Positive Political Economy, edited by James Alt and Kenneth Shepsle. Cambridge University Press.

Lazear, Edward P. (1995). "Corporate Culture and the Diffusion of Values." In Trends in Business Organization, edited by Horst Siebert. J.C.B. Mohr.

Mas-Colell, Andreu, Michael D. Whinston, Jerry R. Green (1995). Microeconomic Theory. Oxford University Press.

Milgrom, Paul R., and John Roberts (1995). "Complementarities and Fit: Strategy, Structure and Organizational Change in Manufacturing." Journal of Accounting and Economics, 19, 179-208.

Mirza, Daniel, and Giuseppe Nicoletti (2004). "What is So Special About Trade in Services." Research Paper No. 2004/02, University of Nottingham.

Oulton, Nicholas (2000). "A Tale of Two Cycles: Closure Downsizing and Productivity Growth in Manufacturing, 1973-1989." National Institute Economic Review, 173, 66-79.

Radner, Roy (1993). "The Organization of Decentralized Information Processing." Econometrica, 61, 1109-1146.

Reynolds, Winston A. (1959). "The Burning Ships of Hernán Cortés.” Hispania, 42, 317-324.

Santos-Pinto, Luis, and Joel Sobel (2005). "A Model of Positive Self-Image in Subjective Assessments." American Economic Review, 95, 1386-1402.

Schelling, Thomas (1960). The Strategy of Conflict. Harvard University Press.

Theorell, Töres, Gabriel Oxenstierna, Hugo Westerlund, Jane Ferrie, Jan Hagberg, and Lars Alfredsson (2003). "Downsizing of Staff is Associated with Lowered Medically Certified Sick Leave in Female Employees." Occupational and Environmental Medicine, 60, e9.

Van Damme, Eric (1989). "Stable Equilibria and Forward Induction." Journal of Economic Theory, 48, 476-496.

Van Huyck, John, Raymond C. Battalio, and Richard O. Beil (1993). "Asset Markets as an Equilibrium Selection Mechanism: Coordination Failure, Game Form Auctions, and Forward Induction." Games and Economic Behavior, 5, 485-504. 
van Zandt, Timothy (1999). "Real-Time Decentralized Information Processing as a Model of Organizations with Boundedly Rational Agents." Review of Economic Studies, 66, 633-658. Vives, Xavier (2005). "Complementarities and Games: New Developments." Journal of Economic Literature, 43, 485-504.

Winter, Eyal (2004). "Incentives and Discrimination." American Economic Review, 94, 764-773. 